\title{
Acute pain management in children with sickle cell anaemia during emergency admission to a teaching hospital in Lagos, Nigeria
}

\author{
K A Oshikoya, ${ }^{1} \mathrm{MBBS}, \mathrm{MSc}, \mathrm{PhD}$; B Edun, ${ }^{2} \mathrm{BSc}$; I A Oreagba, ${ }^{2}$ BPharm, $\mathrm{MSc}, \mathrm{PhD}$ \\ Pharmacology Department, Lagos State University College of Medicine, Ikeja, Lagos, Nigeria \\ ${ }^{2}$ Pharmacology, Therapeutics and Toxicology Department, College of Medicine University of Lagos, Idiaraba, Lagos, Nigeria
}

Corresponding author: K A Oshikoya (kazeemoshikoya@ymail.com)

\begin{abstract}
Background. Optimal pain management in children with sickle cell anaemia (SCA) begins with accurate and thorough pain assessment. However, little or no evidence of this practice exists among SCA patients in developing countries.

Objectives. To evaluate pain management in children with SCA during emergency admission.

Methods. Children with SCA who were $\leq 12$ years old, on admission for a painful crisis and who were not using herbal remedies for pain relief, were prospectively studied at the Lagos University Teaching Hospital (LUTH) over a period of 1 year. A proforma data collection form was used to obtain information about the demographics of the patients and their parents, as well as pain score, and non-pharmacological and pharmacological treatments documented in the patients' case files. Pain was assessed for each patient using the Wong-Baker Faces Pain Rating Scale.

Results. The median age and weight of the patients were 4 years and $16 \mathrm{~kg}$, respectively. Pain assessment by the physicians was documented in $10(8.3 \%)$ patients. However, among those assessed for pain by the researchers, 90 (75\%) had a moderate pain score of $5-8$. Fluid therapy $(n=110,91.7 \%)$ and prayers $(n=120,100 \%)$ were the most common non-pharmacological therapies administered to the patients while admitted. Analgesics, either in combinations or as a single medicine, were administered to 100 (83.3\%) patients. Paracetamol ( $n=90,75 \%)$ and pentazocine $(n=80,66.7 \%)$ were the most frequently used analgesics. The dosage of the analgesics prescribed did not conform to the recommendations of the World Health Organization (WHO).

Conclusion. The SCA children in this study were managed sub-optimally with analgesics. Pain management among this group of children did not fully conform to the guidelines of the WHO.
\end{abstract}

S Afr J Child Health 2015;9(4):119-123. DOI:10.7196/SAJCH.2015.v9i4.968

Sickle cell anaemia (SCA) is an incurable genetic disorder widespread in sub-Saharan Africa and among African descendants worldwide. It is an inherited disorder that is characterised by genetic abnormalities and sickling of the red blood cells. ${ }^{[1]}$ According to the World Health
and Organization (WHO), over 300000 babies with severe haemoglobin disorders are born each year, with Nigeria accounting for about three-quarters of these births. ${ }^{[2]}$ Sixty percent of these children die as infants.

Most SCA children are ambulatory and often enter the acute inpatient system for debilitating events such as crises or acute pain. Crippling pain is the most frequent symptom associated with SCA. ${ }^{[3]}$ It can be unrelenting, unpredictable and vary from acute to chronic or mixed. Acute pain is the leading cause of emergency department visits and hospitalisations among children with SCA in the USA. ${ }^{[4]}$ It is an episodic pain that is primarily due to tissue ischaemia and vaso-occlusion of the microcirculation by sickled cells. ${ }^{[5]}$ Frequently, it acts as a protective agent in response to tissue injury, thereby warning the body of the presence of potentially harmful agents. ${ }^{[6]}$ Just as the injury heals, the pain disappears. The resolution of acute pain usually occurs in a few days to several weeks. ${ }^{[7]}$ The arms, legs, abdomen, chest and back are the most common locations of pain episodes. Acute pain associated with SCA children is often described as aching, tiring and uncomfortable. This may be experienced as early as $6-12$ months of age.

One of the most debilitating aspects of vaso-occlusive episodes is their unpredictable nature in terms of frequency, intensity, affected sites and duration of pain. ${ }^{[5]}$ It is thought that vaso-occlusive episodes are triggered by various environmental and psychological states, such as high altitudes, extreme temperatures, infection, dehydration, stress and fatigue ${ }^{[8]}$ Painful episodes experienced by children with SCA often interfere with intellectual activities, such as attending school and completing homework, social activities such as participating in activities with family members and peers, and the quality and quantity of sleep. Currently, there are many means of managing pain in children with SCA, which include pharmacological and nonpharmacological approaches. ${ }^{[4]}$ However, the effectiveness of the approaches has not been extensively reported in the literature.

The WHO has developed guidelines for pain management in children. ${ }^{[9]}$ Pain management may, however, vary from one country to another ${ }^{[10,11]}$ as well as from one institution to another. ${ }^{[12]}$ The Standard Treatment Guidelines in Nigeria contain a step-by-step approach to managing acute pain in SCA, which is helpful for clinicians who care for children with pain crises. ${ }^{[13]}$ Generally, the guidelines deal specifically with the pharmacological and nonpharmacological management of persisting (long-term) pain in children with medical illnesses, as well as those with SCA.

Optimal pain management begins with an accurate and a thorough pain assessment. Pain assessment enables healthcare providers to treat pain and alleviate needless suffering. It should be carried out at regular intervals because the disease process and the factors that influence it may change over time, and regular assessment permits the measurement of the efficacy of different treatment strategies in relieving pain. The pain assessment process involves the child and their parents or caregivers, and the healthcare providers. Pain 
assessment is, therefore, an integral part of the clinical care of SCA children.

Holistic treatment of pain includes use of opioids, ${ }^{[14]}$ non-opioids and adjuvant therapy that includes cognitive behavioural therapy and psychotherapy. ${ }^{[15]}$

In order to avoid poor or inadequate treatment of children in pain, it is essential for clinicians to adequately assess the pain history and individual patient's needs. However, one of the challenges for healthcare providers in treating pain in SCA is that there is no objective method for assessing underlying vaso-occlusive pathophysiological alterations directly. ${ }^{[16]}$ Pain history is determined by self-report and there are usually no obvious biophysical signs.

A dearth of information on the manner in which clinicians manage acute pain in SCA children in developing countries necessitated this study. Therefore, our objective was to evaluate acute pain management in SCA children on admission to the emergency room in a typical Nigerian teaching hospital.

\section{Methods}

According to the 2006 National Census, ${ }^{[17]}$ Lagos State has an estimated population of over 17 million. Lagos is served by two teaching hospitals: the Lagos University Teaching Hospital (LUTH) and Lagos State University Teaching Hospital (LASUTH). LUTH is funded by the Federal Government of Nigeria while LASUTH is funded by the Lagos State Government. Both hospitals serve as major referral centres to the surrounding health facilities in South Western Nigeria and beyond because of the facilities and the high standard of care that is available.

This was a single-centre prospective study conducted at LUTH, IdiAraba, Surulere, Lagos state, South-West Nigeria between 1 January and 31 December 2014. The study involved children with SCA who were $\leq 12$ years old, on admission for a painful crisis and not using herbal remedies for pain relief.

A proforma data collection sheet, purposively designed for this study, was used to obtain information about the demographics of the children (age, gender, weight) and their parents/caregivers (religion, and marital and employment status). Other sections of the proforma were used to obtain information on the duration of admission of the patients, pain score assessment by the physician before and after analgesic use, and non-pharmacological and pharmacological treatments documented in the patient's case file. One of the researchers assessed each patient for pain using the Wong-Baker Faces Pain Rating Scale. ${ }^{[18]}$ Detailed information about the medicines used during admission was also obtained from the case files of the patients. Attention was paid specifically to the type, dosage, duration and frequency of use, and route of administration of the analgesics administered to the patients. The dosages of the various analgesics used were compared with those recommended for children by the $\mathrm{WHO}^{[9]}$ or Drugs.com (http://www.drugs.com/dosage/). ${ }^{[19]}$ This enabled us to grade the dosages as normal, under or over.

The study protocol was approved by the Ethics Committee of LUTH. Data were analysed using SPSS version 13 (IBM, USA) and presented as simple descriptive statistics and pictograms.

\section{Results}

A total of 120 SCA patients were admitted during the period of study and constituted the population of patients studied. The median age and weight of the children were 4 years and $16 \mathrm{~kg}$, respectively (Table 1). Heights were not recorded in their case files. All the parents were married and employed in a job.

Pain scoring by the physicians was done in only 10 (8.3\%) patients. Unfortunately, the pain assessment tools used by the physicians were not documented in the case files of these patients. However, one of the current study researchers conducted pain assessment on the patients on the same day $(n=90,75 \%)$, day $1(n=20,16.7 \%)$, and day $2(n=10$,
$8.3 \%$ ) of admission (Table 2). Most of the patients $(n=90,75 \%)$ had moderate pain scores of 5 - 8 on the day of admission.

Fluid therapy $(n=110,91.7 \%)$ and prayers $(n=120,100 \%)$ were the only non-pharmacological therapy administered to the patients during emergency admission.

Antibiotics were used, either in combination or as a single medicine, for $80(66.7 \%)$ patients. The antibiotics used were

Table 1. Demographics of children with sickle cell anaemia and their parents

\begin{tabular}{ll}
\hline Demographics & Values $(\mathbf{N = 1 2 0})$ \\
\hline Age (years), Q1 - Q3 (median) & $3-9(4)$ \\
Weight (kg), Q1 - Q3 (median) & $12-20(16)$ \\
Religion, $n(\%)$ & \\
$\quad$ Christianity & $110(91.7)$ \\
Islam & $10(8.3)$ \\
Marital status of parents (married), $n(\%)$ & $120(100)$ \\
Employment status of parent (employed), $n(\%)$ & $120(100)$
\end{tabular}

Table 2. Pain assessment in children with sickle cell anaemia in painful crises as determined by the researchers

\begin{tabular}{lll}
\hline Day of admission & Pain score & $\boldsymbol{n}(\%)$ \\
\hline Same day & 5 & $20(16.7)$ \\
& 6 & $30(25.0)$ \\
& 7 & $20(16.7)$ \\
& 8 & $20(16.7)$ \\
Day 1 & 2 & $10(8.3)$ \\
& 3 & $10(8.3)$ \\
Day 2 & 1 & $10(8.3)$ \\
${ }^{*}$ Pain score, based on the assessment tool, ranges from 1 to 10. &
\end{tabular}

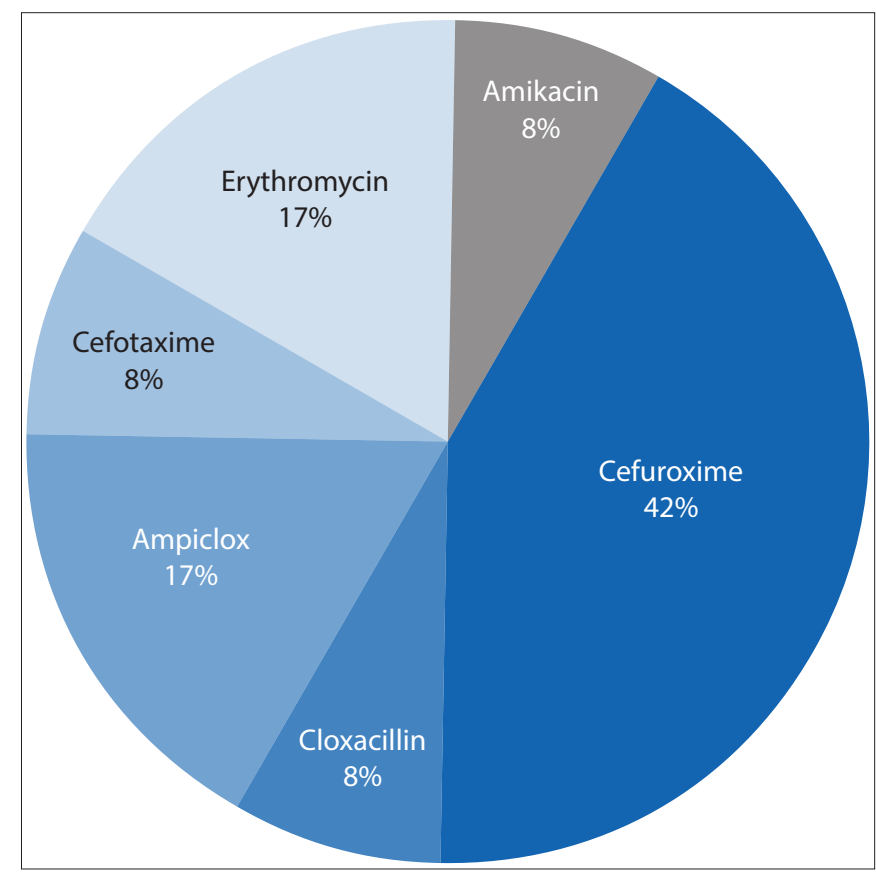

Fig. 1. Pattern of antibiotics used for children with SCA admitted for painful crises. 
erythromycin, amikacin, cefuroxime, cefotaxime, ampicillin with cloxacillin (ampiclox), and cloxacillin alone (Fig. 1). Cefuroxime $(n=50,41.7 \%)$ was the most frequently used antibiotic recorded during the study.

Analgesics, either in combinations or as a single medicine, were administered to $100(83.3 \%)$ patients. Figure 2 shows the pattern of analgesics administered to the patients during acute pain management. Paracetamol $(n=90,75 \%)$ and pentazocine $(n=80$, $66.7 \%$ ) were the most frequently used analgesics (Fig. 2). Morphine and its derivatives, except hydromorphone, were rarely used.

Among the 90 SCA children prescribed paracetamol, $11.1 \%$ were prescribed a normal dose of $10-15 \mathrm{mg} / \mathrm{kg} /$ day, 33.3\% were underdosed, and $55.6 \%$ were overdosed. Paracetamol was prescribed either intramuscularly or intravenously in 3 - 4 divided doses per day, and the duration of use was left open until the pain had adequately subsided. Among the 80 patients prescribed pentazocine, $75 \%$ were prescribed a normal dose of $0.5-1 \mathrm{mg} / \mathrm{kg} / \mathrm{day}$, none were underdosed and $25 \%$ were overdosed. After an intramuscular starting (statim) dose of pentazocine, it was subsequently administered when necessary (pro re nata). Among the 70 patients prescribed ibuprofen, $57.1 \%$ were prescribed a normal dose of $4-10 \mathrm{mg} / \mathrm{kg} / \mathrm{day}$, none were underdosed and $42.9 \%$ were overdosed. Ibuprofen was prescribed orally in three divided doses per day.

The analgesics prescribed were among those recommended by the WHO (Table 3). However, some of the individual doses of the analgesics administered were not in line with the WHO guidelines. A few patients $(n=10,8.3 \%)$ were treated for acute malaria with artesunate. Prophylactic use of proguanil was observed in six patients. The dosage of proguanil prescribed was $50 \mathrm{mg}$ daily and was given orally. Only $40(30.3 \%)$ patients were administered medicines other than antimalarials, antibiotics and analgesics. Sedatives or tranquilisers were not administered to any of the patients. However, folic acid (33\%), hydroxyurea (17\%), furosemide (17\%) and zinc

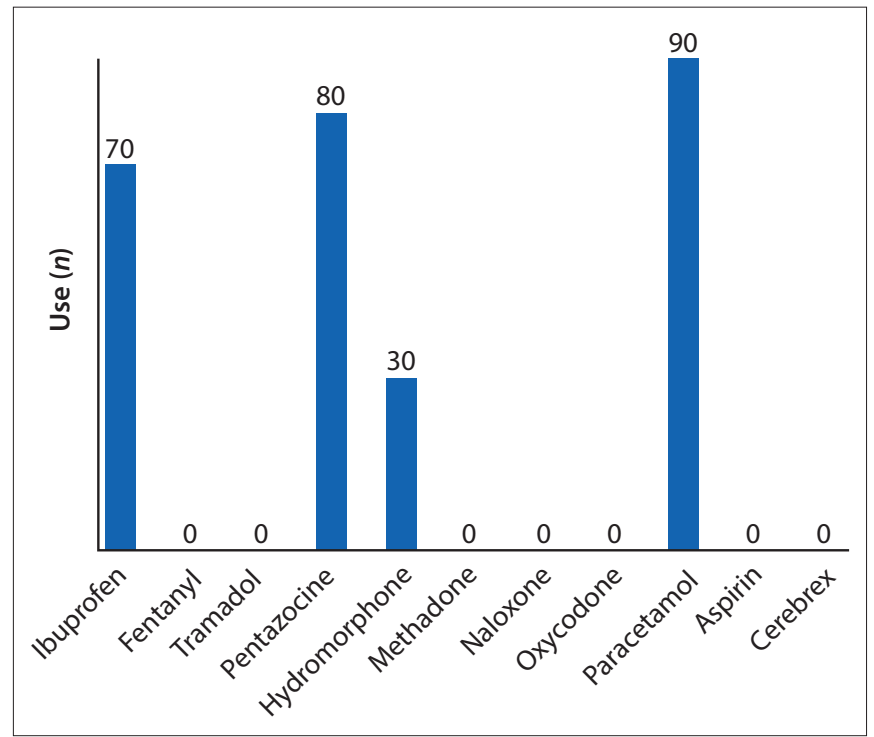

Fig. 2. Analgesics used for children with SCA admitted for acute pain crises.
(17\%), were administered as adjunct therapies to analgesics during the acute pain management (Fig. 3).

\section{Discussion}

Anthropometric parameters such as the age, weight and height are important in calculating or estimating drug dosages for children. Unfortunately, only the age and weight of the patients were documented in their case files. The implication of this is that the patients are at risk of over- or underdosing with analgesics and other adjunct medicines. Overdosing with medications could result in drug toxicity or adverse events, while underdosing could result in undertreatment, persistent pain and prolonged hospitalisation. ${ }^{[20]}$

According to the World Health Organization (WHO), ${ }^{[9]}$ initial pain assessment of a child should include a detailed pain history, a physical examination, the diagnosis of the causes, and the measurement of pain severity using an age-appropriate pain measurement tool. Unfortunately, only a few of the guidelines were followed. Pain on admission was assessed by the physicians in only $8.3 \%$ of the SCA patients. The assessment was rather subjective since the tool used was not documented. It is imperative for the attending physician to ask and document what pain treatments have been used previously by the patients, as well as the efficacy of the treatments. Surprisingly, this was not documented in the case files, if ever asked.

One-time pain assessment was performed on a few of the patients by the physicians, which is grossly inadequate. Pain assessment should be performed at regular intervals ( $2-4$ hourly) during the implementation of the pain management plan. ${ }^{[9]}$ This permits the measurement of changes in the severity of pain over time, and the assessment of the adequacy and efficacy of the chosen treatment, enabling adjustments to be made, as necessary.

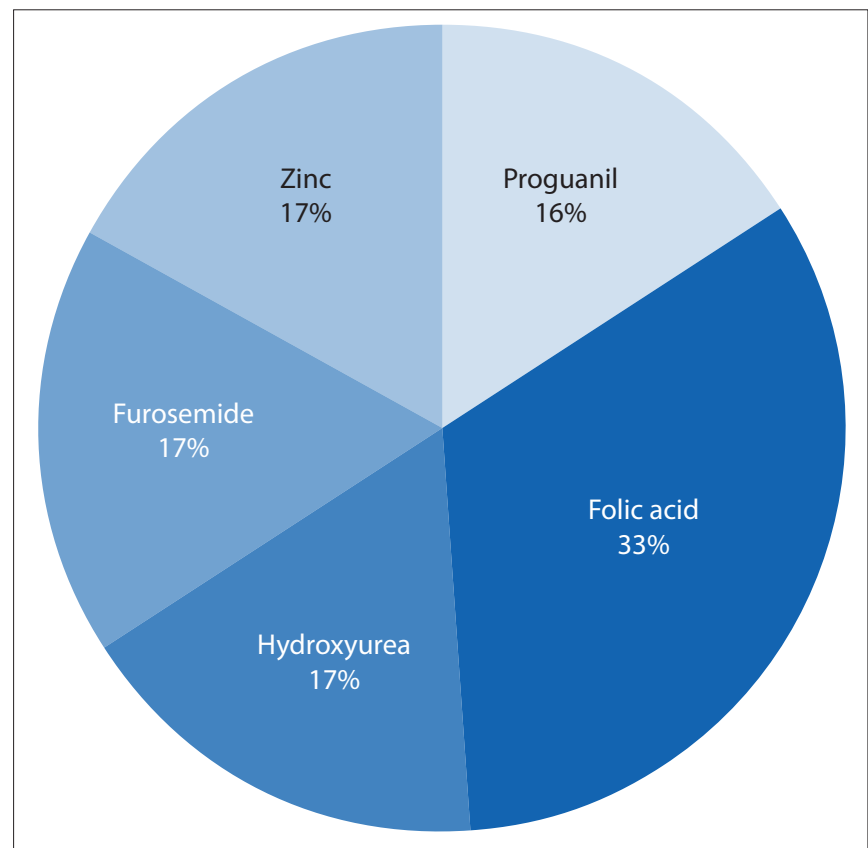

Fig. 3. Adjunct pharmacotherapy co-administered to children with SCA admitted for acute pain crises.

Table 3. Adherence to the WHO medicine and dosage guidelines for pain management in children

\begin{tabular}{lllll}
\hline Type of analgesic & WHO recommended dosage $(\mathrm{mg} / \mathrm{kg})$ & Underdosing $(\%)$ & Overdosing $(\%)$ & Normal dosing $(\%)$ \\
\hline Ibuprofen & $4-10$ & - & 42.9 & 57.1 \\
Paracetamol & $10-15$ & 33.3 & 55.6 & 11.1 \\
Pentazocine & $0.5-1.0$ & - & 25.0 & 75.0
\end{tabular}


The assessment of pain in the SCA patients on the day of admission, using the visual analogue scale, showed that $75 \%$ had a moderate pain score of 5 to 8 . Following this assessment, a detailed pain management plan, including pharmacological and non-pharmacological interventions, can be formulated and implemented. However, a lack of detailed pain assessment by the physicians attending to the SCA patient on admission may compromise adequate and appropriate pain management.

Non-pharmacological approaches for the treatment of pain in children include psychological strategies, education and parental support. For SCA children in pain crises, cognitive-behavioural therapy interventions, which decrease anxiety and distress, are known to be quite effective. ${ }^{[21]}$ The objective of non-pharmacological therapies is to provide responses that may help children master a distressing situation, ideally in a manner consistent with their basic coping strategies. Most of these techniques take time to learn and master, so simple distraction techniques that divert attention away from painful stimuli, or positive incentive techniques that provide a small reward such as prizes for attempts at mastery of their responses, can be effective for children with mild pain. Fluid therapy and prayers were the only non-pharmacological therapies used in the patients in this study. While the former was prescribed and administered by physicians, the latter was provided by the parents of the SCA patients. The role of prayer in alleviating pain in children has not been proven scientifically, while fluid therapy tends to decrease the cellular sickle cell (HbSS) concentration by causing hypotonic swelling of erythrocytes and decreased sickling. ${ }^{[6,10]}$

Antibiotics, either in combination or as a single medicine, were used in $\sim 60 \%$ of the patients; cefuroxime, a first-generation cephalosporin was the most frequently used. These medicines were used empirically for treating presumptive sepsis and in line with the recommended guidelines for pain management in SCA. ${ }^{[10]}$ In a landmark study of 30 participating centres, ${ }^{[2]}$ specific causes of pain were identified in $38 \%$ of patients, and were infections in $29 \%$ of cases. Infections were equally distributed between bacterial, viral, mycoplasma and chlamydial infections, suggesting potential treatment with macrolide and cephalosporin antibiotics. A painful vaso-occlusive crisis may also be associated with fever. Since increased susceptibility to both Gram-positive and Gram-negative organisms characterises this patient cohort, infection and vaso-occlusion might coexist, such that rigorous assessment and initiation of empirical antibiotic treatment is routine, pending the time of availability of culture results. ${ }^{[22]}$ Treating infection alone is known to significantly decrease pain in SCA patients. ${ }^{[23]}$

Analgesics, either in combinations or as a single medicine, were prescribed for almost all the SCA patients during admission for pain crises. Paracetamol and ibuprofen are the medicines of choice recommended by the WHO in the first step of management of mild pain in children. ${ }^{[9]}$ This may be the reason why paracetamol was prescribed for $75 \%$ of the patients in our study. However, the majority of the patients were assessed by the researchers to have moderate pain, which requires a stronger and more effective analgesic than the paracetamol administered for first-line pain relief to the patients. This would suggest inadequate treatment of pain in SCA children.

Moderate or severe pain requires the use of a stronger opioid analgesic. Morphine is the medicine of choice for this kind of pain, although other opioids can be considered and made available to ensure an alternative to morphine in cases of unpleasant sideeffects. ${ }^{[9]}$ Unfortunately, despite the moderate nature of the pain experienced by our patients, morphine and its derivatives - except pentazocine and hydromorphone - were rarely prescribed. This may be due to the problem of unavailability and high cost. It may also be related to certain misconceptions surrounding pain management in children, which can lead to poor treatment outcomes. ${ }^{[9,10]}$ Such misconceptions include the myth that infants and children do not feel pain or suffer less than adults, fears of respiratory depression or other adverse effects of analgesic medications, and the belief that preventing pain in children takes too much time and effort.

Most of the pain medications were administered intramuscularly which is contrary to all guidelines on pain management in children. This is poor practice and should be discouraged. It is therefore imperative to adopt a multidisciplinary approach to managing pain in Nigerian children, which should involve a paediatric clinical pharmacologist with a bias for pain management. Where this expertise is scarce, guidelines for pain management and judicious analgesic use should be developed and properly implemented in our hospitals.

Underdosing (33.3\%) and overdosing (55.6\%) with paracetamol was observed in the present study. However, with pentazocine $(25.0 \%)$ and ibuprofen (42.9\%), only overdosing errors were observed. While paracetamol underdosing could result in poor pain control in SCA children, overdose could cause supratherapeutic toxicity. ${ }^{[24]}$ Children may be at risk of gastrointestinal bleeding, impaired liver and renal function, and metabolic disorders following ibuprofen overdose. ${ }^{[25}$ However, none of the patients manifested any of these adverse events during the study.

Antimalarial drugs and hydroxyurea are among the adjunct treatments received by the patients. Patients with SCA are more vulnerable to malaria because of their weakened immunity. Malaria, like infection, can produce fever, which is associated with a painful vaso-occlusive crisis. ${ }^{[10]}$ Treating malaria fever will, to an extent, indirectly reduce vaso-occlusive painful crises. This justifies the need for antimalarial use by SCA patients.

This study was characterised by some limitations, such as the use of a small sample size. Biases can result from data obtained from a small sample size; therefore, caution is required in interpreting our results. The study was limited to a single centre in Nigeria. Different results may be obtained if a similar study was conducted in other centres. It is, therefore, difficult to generalise our results. Thus, a multicentre study is required to give a true picture of acute pain management in SCA children in Nigeria.

\section{Conclusion}

SCA children admitted for acute pain were managed suboptimally with analgesics. The percentage compliance to the WHO analgesic dosing guidelines varied with different types of analgesics, especially with paracetamol. Overdosing errors were more prevalent than underdosing errors with the three most frequently used analgesics in this study. Educational intervention as well as training and retraining of the physicians involved in pain management of children with SCA is recommended as a means of improving acute pain management.

\section{References}

1. Bunn FH. Pathogenesis and treatment of sickle cell disease. N Engl J Med 1997;33(11):762-769

2. World Health Organization (WHO). Fact Sheet No. 308, 2011. Sickle-cell disease and other haemoglobin disorders. http://www.who.int/mediacentre/ factsheets/fs308/en/ (accessed 4 January 2015).

3. Edwards CL, Scales MT, Loughlin C, et al. A brief review of the pathophysiology, associated pain, and psychosocial issues in sickle cell disease. Int J Behav Med 2005;12(3):171-179. [http://dx.doi.org/10.1207/s15327558ijbm1203_6]

4. Elander J, Lusher J, Bevan D, et al. Pain management and symptoms of substance dependence among patients with sickle cell disease. Soc Sci Med 2003;57(9):1683-1696. [http://dx.doi.org/10.1016/S0277-9536 (02)00553-1]

5. Ballas SK. Sickle cell disease: Clinical management. Baillieres Clin Haematol 1998;11(1):185-214

6. Okpala I, Thomas V, Westerdale N, et al. The comprehensive care of sickle cell disease. Eur J Haematol 2002;68(3):157-162. [http://dx.doi.org/10.1034/j.16000609.2002.01523.x]

7. Todd KH, Ducharme J, Choiniere M, et al. Pain in the emergency department: Results of the pain and emergency medicine initiative (PEMI) multicentre study. J Pain 2007;8(6):460-466. [http://dx.doi.org/10.1016/j.jpain.2006.12.005]

8. Hofmann M, de Montalembert M, Beauquier-Maccota B, et al. Posttraumatic stress disorder in children affected by sickle-cell disease and their parents. Am J Haematol 2007;82(2):171-172. [http://dx.doi.org/10.1002/ajh.20722] 
9. World Health Organization (WHO). WHO guidelines on the pharmacological treatment of persistent pain in children with medical illnesses. WHO 2012. http:// whqlibdoc.who.int/publications/2012/9789241548120_Guidelines.pdf?ua=1 (accessed 2 March 2015)

10. Rees DC, Olujohungbe AD, Parker NE, et al. Guidelines for the management of the acute painful crisis in sickle cell disease. Br J Haematol 2003;120(5):744-752.

11. Marlowe KF, Chicella MF. Treatment of sickle cell pain. Pharmacotherapy 2002;22(4):484-491. [http://dx.doi.org/10.1592/phco.22.7.484.33675]

12. Zempsky WT. Evaluation and treatment of sickle cell pain in the emergency department: Paths to a better future. Clin Pediatr Emerg Med 2010;11(4):265273. [http://dx.doi.org/10.1016/j.cpem.2010.09.002]

13. The Federal Ministry of Health, Nigeria. Standard Treatment Guidelines, Nigeria, 2008. http://apps.who.int/medicinedocs/documents/s17035e/s17035e. pdf (accessed 27 February 2015)

14. Jacob E, The American Pain Society. Pain management in sickle cell disease. Pain Manag Nurs 2001;2(4):121-131.

15. Thomas VJ, Taylor LM. The psychosocial experience of people with sickle cell disease and its impact on quality of life: Qualitative findings from focus groups. Br J Health Psychol 2002;7(Part 3):345-363. [http://dx.doi. org/10.1348/135910702760213724]

16. Todd KH, Green C, Bonham VL, et al. Sickle cell disease related pain Crisis and conflict. J Pain 2006;7(7):453-458. [http://dx.doi.org/10.1016/j. jpain.2006.05.004]
17. National Population Commission. 2006 Population and Housing Census of the Federal Republic of Nigeria. National and State Population and Housing Tables. Priority Tables, Volume 1. http://www.population.gov.ng/images/Priority\%20 Tables\%20Volume\%20I-update.pdf (accessed 17 January 2015).

18. Wong-Baker Faces Foundation. Wong-Baker FACES Pain Rating Scale. http:// wongbakerfaces.org/ (accessed 2 December 2014).

19. Drugs.com. Drug dosage. http://www.drugs.com/dosage/ (accessed 2 December 2014)

20. Oshikoya KA, Ojo OI. Medication errors in paediatric outpatient prescriptions of a teaching hospital in Nigeria. Nig Q J Hosp Med 2007;17(2):74-78.

21. Haywood C, Beach MC, Lanzktron S, et al. A systematic review of barriers and interventions to improve appropriate use of therapies for sickle cell disease. J Nat Med Assoc 2009;101(10):1022-1033.

22. Vichinsky EP, Neumayr LD, Earles AN, et al. Causes and outcomes of the acute chest syndrome in sickle cell disease. N Engl J Med 2000;342(25):1855-1865. [http://dx.doi.org/10.1056/NEJM200006223422502]

23. Benjamin L, Swinson G, Nagel R. Sickle cell anaemia day hospital: An approach for management of uncomplicated painful crisis. Blood 2000;95(4):1130-1136.

24. Daly FF, O'Malley GF, Heard K, et al. Prospective evaluation of repeated supratherapeutic acetaminophen (paracetamol) ingestion. Ann Emerg Med 2004;44(4):393-398. [http://dx.doi.org/10.1016/S0196064404005244]

25. Drugs.com. Ibuprofen side effects. http://www.drugs.com/sfx/ibuprofen-sideeffects.html (accessed 2 December 2014). 